\title{
The variability classes in IGR J17091-3624 during the 2011 outburst $^{\star}$ (Research Note)
}

\author{
Z. Zhang ${ }^{1,2}$, J. L. Qu ${ }^{1}$, H. Q. Gao ${ }^{1,2}$, S. Zhang ${ }^{1}$, Q. C. Bu ${ }^{3}$, M. Y. Ge ${ }^{1}$, L. Chen ${ }^{3}$, and Z. B. $\mathrm{Li}^{4}$ \\ 1 Key Laboratory for Particle Astrophysics, Institute of High Energy Physics, Chinese Academy of Sciences (CAS), \\ 19B Yuquan Road, 100049 Beijing, PR China \\ e-mail: zhangzhen@ihep.ac.cn \\ 2 University of Chinese Academy of Sciences, 19A Yuquan Road, 100049 Beijing, PR China \\ 3 Department of Astronomy, Beijing Normal University, 100875 Beijing, PR China \\ ${ }^{4}$ Xinjiang Astronomical Observatory, CAS, 150, Science 1-Street, Urumqi, 830011 Xinjiang, PR China
}

Received 20 December 2013 / Accepted 24 July 2014

\begin{abstract}
Aims. Based on the Rossi X-ray Timing Explorer (RXTE) data, we studied the typical variability classes in IGR J17091-3624 during the 2011 outburst and attempted to understand the physical mechanism that produces the highly structured variability.

Methods. Using the rate-resolved spectroscopy, we produced color-color diagrams (CCDs), hardness-intensity diagrams (HIDs), and rate-resolved energy spectra for each class. The time lags were calculated by means of the cross-power spectrum (CPS) analysis.

Results. Our study provides a comprehensive understanding of the $v, \rho, \alpha, \beta / \lambda, \mu$ and $\gamma$ classes during this outburst. The source shows up with different track directions in the CCD loops and different types of the lag at $\sim 1-6 \mathrm{~s}$ which are class dependent. It is notable that each variability class showing hard lags presents an anti-clockwise loop in the CCD, while each variability class showing soft lags presents a clockwise loop in the CCD. We discuss these observational results with the current models proposed for this type of system. The observed hardness ratio shows an increasing trend toward the high disk temperature for different classes, which can be regarded as evidence for thermal-viscous instability.
\end{abstract}

Key words. stars: individual: IGR J17091-3624 - X-rays: binaries - accretion, accretion disks - black hole physics

\section{Introduction}

The object IGR J17091-3624 is the second black-hole X-ray binary after GRS 1915+105, which has shown a bewildering variety of X-ray light curve patterns (Altamirano et al. 2011). These patterns are usually called "variability classes" and characterized mostly with the large variabilities in amplitude and structure. Some variability classes have presented a loop-like behavior in their color-color diagrams (CCDs) and hardness-intensity diagrams (HIDs; Belloni et al. 1997a, 2000; Altamirano et al. 2011). The loop-like behaviors need to be studied to resolve the issue of whether there are differences in physical origin between different classes. Using the rate-resolved spectroscopy, Pahari et al. (2013a) extracted energy spectra during the peak and dip intervals of the different classes and found that their spectra are phenomenologically complex. We performed more refined spectral analysis for different classes in IGR J17091-3624. In IGR J17091-3624, the power density spectra (PDS) show a band limited noise component up to a break frequency $v_{\mathrm{b}}$. The frequency $v_{\mathrm{b}}$ most plausibly corresponds to a viscous timescale (Ingram et al. 2009), over which flare may occur frequently (Belloni et al. 1997b). Pahari et al. (2013b) found that different types of lags of $\sim 0.1-1.0 \mathrm{~s}$ were observed in different classes. The lag behaviors around $v_{\mathrm{b}}$ provide a new perspective to our understanding the physical origins of the variability classes.

^ Tables 3 and 4 are available in electronic form at http: //www . aanda.org
Table 1. Observational details of different classes used for the analysis.

\begin{tabular}{cccccc}
\hline \hline$N$ & Obs. ID & MJD & PCUs on & Exposure (s) & Class \\
\hline 1 & O-04-03 & 55644.74 & PCU0, PCU2 & 2783 & $\alpha$ \\
2 & O-05-00 & 55648.21 & PCU1, PCU2 & 2720 & $\nu$ \\
3 & O-07-01 & 55662.83 & PCU0, PCU2 & 3200 & $\rho$ \\
4 & O-08-03 & 55670.81 & PCU1, PCU2 & 2352 & $\beta / \lambda$ \\
5 & O-09-06 & 55676.48 & PCU2 & 3280 & $\mu$ \\
6 & O-37-04 & 55873.84 & PCU2, PCU4 & 1264 & $\gamma$ \\
\hline
\end{tabular}

Notes. The variable $N$ is the number of each observation. The letter $\mathrm{O}$ stands for Obs. 96420-01.

\section{Observations and data analysis}

We examined IGR J17091-3624 in its 2011 outburst using RXTE public archival observations and selected out six typical observations. The observational data was obtained with the Proportional Counter Array (PCA) instruments. These observations represent six variability classes (Altamirano et al. 2011, 2012). They are listed in Table 1. All the opened proportional counter units (PCUs) were used for the data analysis to improve statistics.

For each observation interval, we created a light curve with $1 \mathrm{~s}$ bin in three PHA channel intervals $A=0-14$ (2.0-6.5 keV), $B=15-35(6.5-15.0 \mathrm{keV})$, and $C=36-255$ (15.0-60.0 keV). Using the rate resolved spectroscopy, we got 
A\&A 569, A33 (2014)

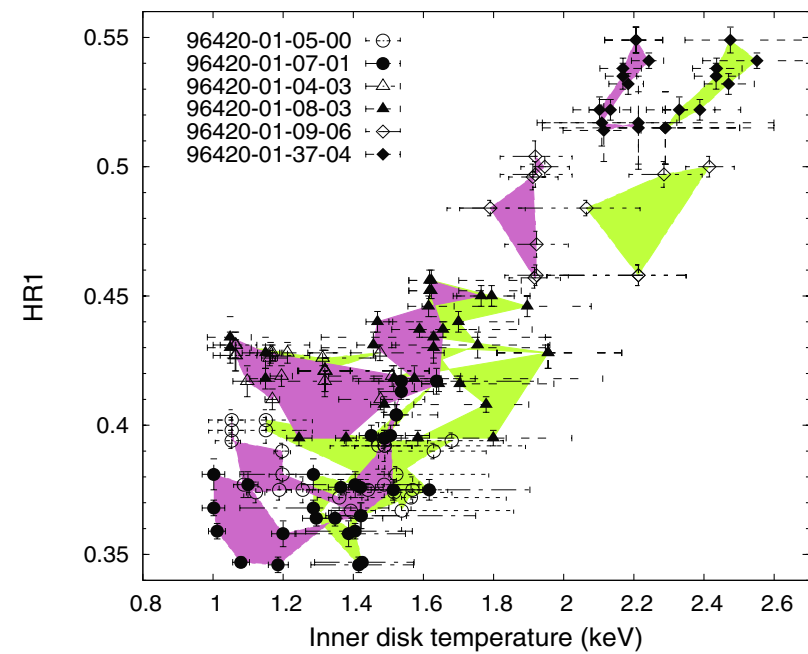

Fig. 1. HR1 vs. $T_{\text {in }}$ for the six typical observations (Tables 3 and 4). Different symbols correspond to different observations. Each color stands for a different disk model. Purple: diskbb. Green: diskpbb.

the spectral parameters from different rate bands. Following Pahari et al. (2013a), we obtained the spectral parameters from the peak and dip, respectively. For those between the peak and dip, we obtained the spectral parameters from the ascending and descending parts, respectively. Flares were separated into bins of $\Delta \mathrm{ct} / \mathrm{s}$ wide intervals, where $\Delta$ is is a given number. From these data, we obtained a total count rate $(R=A+B+C)$ and two hardness values: $\mathrm{HR} 1=B / A$ and HR2 $=C / A$. All the CCDs and HIDs were created by setting $\Delta=50$.

The PDS and light curves were produced from the PCA using standard techniques (e.g., Belloni et al. 2002; Altamirano et al. 2011). The PDS fitting was carried out in a standard way (Belloni et al. 2002). The errors were within $90 \%$ confidence level. The time lags between the high energy intervals (from E1 to E5) and the lowest interval (E0) were quantified by the CPS analysis (Cui et al. 1997). The absolute channels and energy intervals are: E0: 0-8 (2.06-4.09 keV), E1: 9-13 (4.09-6.12 keV), E2: 14-18 (6.12-8.17 keV), E3: 19-23 (8.17-10.22 keV), E4: 24-35 (10.22-15.18 keV) and E5: $36-57$ (15.18-24.85 keV).

Rate-resolved energy spectra were extracted for each observation. All the energy spectra were fit in the 3.0-30.0 keV band using XSPEC version 12.7. The energy spectra have two main components: thermal and non-thermal (Belloni et al. 1997b; Pahari et al. 2013a). Different models can be used to well describe the two components (Pahari et al. 2013a). Following Pahari et al. (2013a), we fit these spectra with different disk models, such as diskbb or diskpbb in addition to the powerlaw model. Some spectra required a Gaussian for good fit. The errors were within $90 \%$ confidence level. To further improve statistic, we perform spectral analysis by setting $\Delta=100$. The hydrogen column density $N_{\mathrm{H}}$ was fixed to $1.1 \times 10^{22} \mathrm{~cm}^{-2}$ (Rodriguez et al. 2011; Krimm et al. 2011).

\section{Results}

In Fig. 1, we plot HR1 versus inner disk temperature $T_{\text {in. }}$. The data points of each class distribute diversely. However, there exists a global relationship between the HR1 and $T_{\text {in }}$ for different classes (Fig. 1). With limited statistics, the HR1 appears to increase with $T_{\text {in }}$, especially when the $T_{\text {in }}$ is higher than $1.2 \mathrm{keV}$.
Table 2. Summary of PDS and lag properties of the six observations.

\begin{tabular}{c|c|cc|cc|c|c}
\hline \hline$N$ & Obs. ID & $\begin{array}{c}v_{\max } \\
(\mathrm{mHz})\end{array}$ & $\begin{array}{c}\operatorname{lag}\left(v_{\max }\right) \\
(\mathrm{s})\end{array}$ & $\begin{array}{c}v_{\mathrm{QPO}} \\
(\mathrm{mHz})\end{array}$ & $\begin{array}{c}\operatorname{lag}\left(v_{\mathrm{QPO}}\right) \\
(\mathrm{s})\end{array}$ & $\begin{array}{c}v_{\mathrm{b}} \\
(\mathrm{mHz})\end{array}$ & $\chi_{\mathrm{red}}^{2}$ \\
\hline 1 & O-04-03 & $15 \pm 5$ & $+1-6$ & $15_{-5}^{+5}$ & $+1-6$ & $80_{-8}^{+9}$ & 0.905 \\
2 & $\mathrm{O}-05-00$ & $25 \pm 5$ & $+1-6$ & $25_{-5}^{+5}$ & $+1-6$ & $116_{-12}^{+12}$ & 0.945 \\
3 & O-07-01 & $50 \pm 10$ & $-0.5-1$ & $50_{-3}^{+11}$ & $-0.5-1$ & $92_{-5}^{+15}$ & 1.031 \\
4 & O-08-03 & $15 \pm 5$ & $-1-6$ & $237_{-9}^{+9}$ & $-0.1-0.2$ & $494_{-59}^{+68}$ & 0.945 \\
5 & O-09-06 & $20 \pm 5$ & $-1-6$ & $392_{-6}^{+5}$ & $-0.1-0.3$ & $225_{-18}^{+18}$ & 0.936 \\
6 & O-37-04 & - & - & - & - & $244_{-43}^{+42}$ & 0.677 \\
\hline
\end{tabular}

Notes. The variable $N$ is the number of each observation (Table 1), $v_{\max }$ denotes the characteristic frequency at which the time lag shows the largest absolute value, and $v_{\mathrm{QPO}}$ denotes the $\sim 10-100 \mathrm{mHz}$ quasiperiodic oscillation (QPO) centroid frequency. The variable $v_{\mathrm{b}}$ denotes the break frequency. $\operatorname{lag}(v)$ is the time lag at a particular frequency $v$.

However, a similar trend is not observed in a HR2- $T_{\text {in }}$ plot (Tables 3 and 4).

Figure 2 shows the CCDs for flares in different classes, where the source does not always follow the same pattern. The source traces out an anti-clockwise loop for the $v$ and $\alpha$ observations and a clockwise loop for the $\mu, \rho$, and $\beta / \lambda$ observations. In Fig. 2, the insets show HIDs of the PCA count rate and the HR1 value (hereafter HID1s). All the HID1 loops are traced in the same direction with the CCD loops. In the $\gamma$ observation, the source does not trace out a CCD loop. In Fig. 3, we plot PCU2 count rate versus HR2 (hereafter HID2s). A strong anticorrelation between PCU2 count rate and HR2 is observed for different classes and their evolutions follow the remarkable similar pattern. Each class shows a single elongated branch stretching out diagonally.

Figure 2 also shows representative PDS. Each one is dominated by a band-limited noise component, which breaks at a frequency $v_{\mathrm{b}}$, below which the spectrum is so strong that the fractional power density is about $0.1 \%$ per hertz (Fig. 2). In the PDS, one observes a $\sim 10-100 \mathrm{mHz}$ QPO $\left(Q=v_{\mathrm{QPO}} /\right.$ full width half maximum $>2$ ) from the "flares". Each quasi-periodic oscillation (QPO) has a centroid frequency $v_{\mathrm{QPO}}$ below or approximately equal to $v_{\mathrm{b}}$ (Table 2). In the right panels of Fig. 2, we present the energy-lag spectra from the frequency bands around $v_{\mathrm{QPO}}$, $v_{\mathrm{b}}$, and $v_{\max }$ (Table 2). The QPOs with $v_{\mathrm{QPO}} \lesssim v_{\mathrm{b}}$ show obvious time lags. For the $\gamma$ observation, even if there are no such QPO features visible in the PDS, we still performed the time lag analysis for the strong noise component at frequency below or approximately equal to $v_{\mathrm{b}}$.

In the $v$ and $\alpha$ observations, the $\sim 10 \mathrm{mHz}$ QPOs show hard lags of $\sim 1-6 \mathrm{~s}$ (Fig. 2 and Table 2 ). In the $\mu, \rho$, and $\beta / \lambda$ observations, the $\sim 10-100 \mathrm{mHz}$ QPOs show soft lags of $\sim 0.1-6 \mathrm{~s}$ (Fig. 2 and Table 2). In the $\mu$ observation, the time-lag spectra show soft lags of $\sim 1-6 \mathrm{~s}$ in the frequency band from $\sim 10 \mathrm{mHz}$ to $v_{\mathrm{b}}$ (Fig. 2). In the $\gamma$ observation, the strong noise component does not show any hints of the time lag around $1-6 \mathrm{~s}$ (Fig. 2). It is notable that the observations observed with $v_{\mathrm{QPO}} \lesssim v_{\mathrm{b}}$ show obvious lags, while the one with no similar QPO feature behaves with a less prominent time lag (Table 2).

For different classes, the largest absolute value of the observed lags tend to occur in different frequency bands (see $v_{\max }$ values in Table 2). For a fixed frequency band, the time lags appear to vary between different energy intervals. As observed in Fig. 2, for example, the hard lags at $v_{\max }$ tend to increase with energy ranging from $\sim 1$ to $\sim 6 \mathrm{~s}$, while the soft lags at $v_{\max }$ tend to decrease with energy ranging from $\sim-1$ to $\sim-6 \mathrm{~s}$. It is clear that 
Z. Zhang et al.: Variability classes in IGR J17091-3624 (RN)
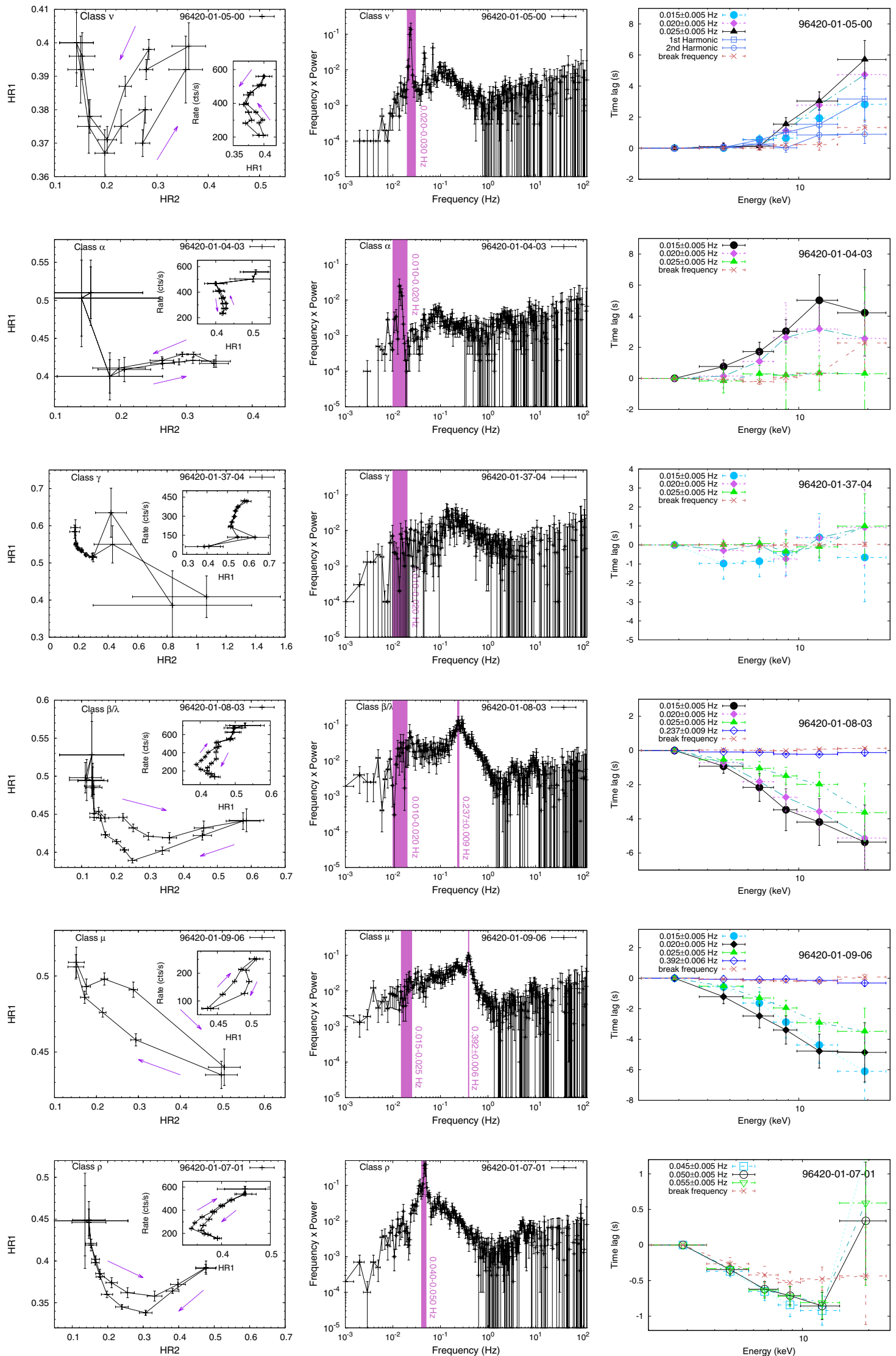

Fig. 2. Left panels: CCDs extracted from the six typical observations. Insets show HID1s. Rates are given in per rate band with all the opened PCUs, 2-60 keV, and background subtracted. Middle panels: PDS. Shaded areas mark the frequency bands around $v_{\mathrm{QPO}}$ and $v_{\mathrm{max}}$. Right panels: time lags as a function of energy for various frequency bands vs. the lowest energy interval $(2.06-4.09 \mathrm{keV})$. Different symbols correspond to different frequency bands. 


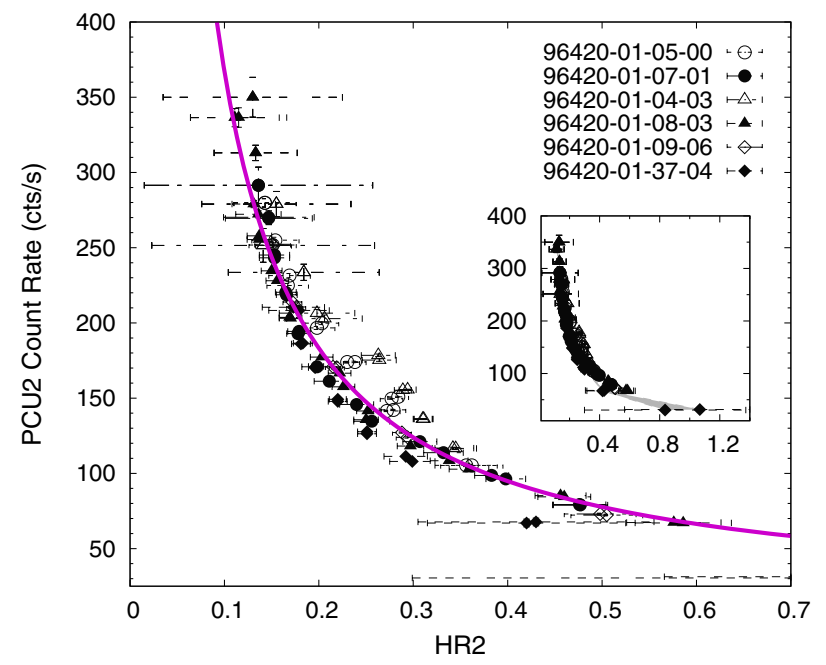

Fig. 3. HIDs of the PCU2 count rate and the HR2 value. Different symbols correspond to different observations. The anticorrelation is well described by a power law of the form $y=a x^{-b}+c$, where $a=30.96 \pm 16.98, b=1.05 \pm 0.25, c=13.22 \pm 29.37$, and $\chi_{\text {red }}^{2}=0.94$.

the time lags of $\sim 1-6 \mathrm{~s}$ depend strongly on photon energy. The higher the energy, the larger the lag timescale (with the exception of the $45 \mathrm{mHz}$ QPO in the $\rho$ observation, where the lag timescale increases at first and then drops above $12 \mathrm{keV}$ ).

We found a strong relation between the CCD and the time lag: the observations showing hard lag features present a CCD loop traced out in an anti-clockwise direction while the observations showing soft lag features present a CCD loop traced out in a clockwise direction. It suggests an intrinsic relation between the track direction of the CCD loop and the sign of the time lag.

\section{Discussion and conclusions}

Belloni et al. (1997b) interpreted the large variability of the X-ray flux of GRS $1915+105$ with the rapid removal and replenishment of matter, which forms a hot accretion flow and is driven by the thermal-viscous instability. Since IGR J17091-3624 and GRS $1915+105$ were observed with similar behavior, it is reasonable to speculate that both have a similar physics underneath (Capitanio et al. 2012). In this model, the source evolves in form of loops between the lower and upper branches of the S-shaped $\dot{M}(\Sigma)$-curve (Belloni et al. 1997b). Each loop refers to a single cycle of instability (Janiuk et al. 2005) and, as suggested in Belloni et al. (1997b), should show up in CCD. In IGR J17091-3624, several classes were observed with the looplike behaviors in the CCD and HID1, which are also due to the cycles of instability. We find for the first time that the track directions of their CCD and HID1 loops are highly class dependent, which is in turn suggestive of having the different intrinsic physical origins.

For different classes, the HR1 shows an increasing trend toward high $T_{\text {in }}$. This global trend suggests that these classes may share with a common physical mechanism. If the variability classes are caused by thermal-viscous instability, it is usually thought that the disk is truncated at a region where $P_{\text {rad }}$ is balanced with $P_{\text {gas }}$ (Belloni et al. 1997b). Here $P_{\text {rad }}$ is the radiation pressure and $P_{\text {gas }}$ is the gas pressure. This region is the innermost part of an accretion flow, according to the description by a standard disk model. Thus, the inner disk temperature can be used to describe the region where $P_{\text {rad }} \sim P_{\text {gas }}$. When the luminosity increases, the disk moves inwards and the hot accretion flow extends toward outside to strengthen the corona, resulting in a harder spectrum and hence a larger hardness ratio (Lei et al. 2013). With the $P_{\text {rad }}$ increasing, $P_{\text {gas }}$ increases because $P_{\text {gas }} \sim P_{\text {rad }}$. Then $T_{\text {in }}$ increases with the increasing $P_{\text {gas }}\left(=n k T_{\text {in }}\right)$. This trend can be regarded as an evidence for the thermalviscous instability.

A similar pattern in the HID2 for the different classes is shown, and those classes showing HID1 loops present no loop in their HID2s. One interpretation is that the thermal-viscous instability become less prominent in the non-thermal dominant inner hot accretion flow with $P_{\text {rad }} \gg P_{\text {gas }}$. Due to the same reason, the HR2 shows no trend of increasing toward higher $T_{\text {in }}$ for different classes.

In IGR J17091-3624, the observed lags are of 1-6s. The timescale of only reflection or the Comptonization process is expected to be about $\lesssim 1 \mathrm{~s}$ (Lei et al. 2013; Pahari et al. 2013b). Therefore, these lags should be related to other process. As mentioned in Janiuk et al. (2005), the hard lag of $\sim 1-6 \mathrm{~s}$ is comparable to the viscous timescale in the hot accretion flow. Such a hard lag is required by the hot accretion flow to adjust to the changing conditions in the disk. However, the model reported in Janiuk et al. (2005) did not explain both energy dependence of the lag and the soft lag. The viscous fluctuation model is also a candidate for an alternative interpretation. In this model, the viscous fluctuations come from outer regions (Pahari et al. 2013b). Since the outer regions typically emits lower energy photons than the inner ones, the expected lag should be at the hard band (Pahari et al. 2013b). When the viscous fluctuations occur at outer regions and propagate inwards, it causes an accretion rate fluctuation in the hot accretion flow, and the expected hard lag from this model should correlate with the energy in a positive manner. However, this viscous propagation model cannot be used to explain the soft lag of $\sim 1$ s (Pahari et al. 2013b). Such a soft lag can be explained when strong disturbance (such as hot gas steams) is produced from the hot accretion flow with $P_{\text {rad }} \sim P_{\text {gas }}$ (Frank et al. 1987) and propagates to the outer and optically thick disk, hence affecting the soft X-ray emission region. In this scenario, the disturbances come from inner regions, which is different from the viscous fluctuation model. The disturbance from the optically thin hot accretion flow contributes to the decreasing of the scattering depth, so that more photons with lower energies can escape from the outer disk. These soft photons escaping from the outer region have a time lag with respect to those hard photons escaping from the inner region. This disturbance from the hot accretion flow perturbs the outer material and then produces the observed soft lag. From this point of view, the soft lag of 1-6s can be regarded as the time taken for the disk to adjust to the disturbance in the hot accretion flow. While the disturbance propagation from the hot accretion flow could manifest itself in a lag observationally, any radial movement of the propagation would cause the lag timescale to increase with energy (Cui et al. 1997). However, it remains unclear how to associate the lag signs with the track directions followed along the CCD.

Acknowledgements. We are thankful to the anonymous referee for providing useful comments/suggestions which improve the paper. This work is supported in part by the National Natural Science Foundation of China (NSFC), XTP project XDA04060604, NSFC-11173024, NSFC-11133002, NSFC-11073021, the NSFC for Young Scientists, grant No. 11203064, and the Strategic Priority Research Program "The Emergence of Cosmological Structures" of the Chinese Academy of Sciences, grant No. XDB09000000. 


\section{References}

Altamirano D., \& Belloni T. 2012, ApJ, 747, L4

Altamirano, D., Belloni, T., Linares, M., et al. 2011, ApJ, 742, L17

Belloni, T., Méndez, M., King, A. R., van der Klis, M., \& van Paradijs, J. 1997a, ApJ, 488, L109

Belloni, T., Méndez, M., King, A. R., van der Klis, M., \& van Paradijs, J. 1997b, ApJ, 479, L145

Belloni, T., Psaltis, D., \& van der Klis, M. 2000, A\&A, 355, 271

Belloni, T., Psaltis, D. \& van der Kils, M. 2002, ApJ, 572, 392

Capitanio, F., Del Santo, M., Bozzo, E., et al. 2012, MNRAS, 422, 3130

Cui, W. 1999, ApJ, 524, L59

Cui, W., Zhang, S. N., Fock, W., \& Swank, J. H. 1997, ApJ, 484, 383
Frank, J., King, A. R., Lasota, J. P. 1987, A\&A, 178, 137

Ingram, A., Done, C., \& Fragile, C. P. 2009, MNRAS, 397, L101

Janiuk, A., \& Czerny, B. 2005, MNRAS, 356, 205

Krimm, H. A., Barthelmy, S. D., Baumgartner, W., et al. 2011, ATel, 3144

Lei, Y. J., Zhang, H. T., \& Zhang, C. M. 2013, ApJ, 146, 60

Pahari, M., Bhattacharyya, S., Yadav, J. S., \& Pandey, S. K. 2012, MNRAS, 422, L87

Pahari, M., Misra, R., Mukherjee, A., Yadav, J. S., \& Pandey, S. K. 2013a, MNRAS, 436, 2334

Pahari, M., Yadav, J. S., Rodriguez, J., et al. 2013b, ApJ, 778, 46

Pahari, M., Yadav, R., \& Bhattacharyya, S. 2014, ApJ, 783, 141

Rodriguez, J., Corbel, S., Caballero, I., et al. 2011, A\&A, 533, L4 
Table 3. Spectral parameters for the different rate bands from the six typical RXTE observations, which are fit with diskbb+powerlaw model.

\begin{tabular}{|c|c|c|c|c|c|c|c|c|}
\hline$\#$ & Obs. ID & $\begin{array}{c}\text { Rate band } \\
\text { (cts/s) }\end{array}$ & HR1 & PL index & $\begin{array}{c}\text { NORM } \\
\text { powerlaw }\end{array}$ & $\begin{array}{c}T_{\text {in }} \\
(\mathrm{keV})\end{array}$ & $\begin{array}{c}\text { NORM } \\
\text { diskbb } \\
\end{array}$ & $\chi_{\mathrm{red}}^{2}$ \\
\hline 1 & O-04-03 & $0-250$ & $0.431 \pm 0.005$ & $2.70_{-0.22}^{+0.20}$ & $0.27_{-0.11}^{+0.17}$ & $1.06_{-0.06}^{+0.06}$ & $11.20_{-4.73}^{+4.35}$ & 1.23 \\
\hline 2 & & $200-300 \mathrm{a}$ & $0.428 \pm 0.004$ & $2.25_{-0.15}^{+0.15}$ & $0.11_{-0.04}^{+0.05}$ & $1.16_{-0.04}^{+0.04}$ & $11.10_{-1.54}^{+1.58}$ & 1.08 \\
\hline 3 & & 200-300d & $0.417 \pm 0.006$ & $2.51_{-0.18}^{+0.17}$ & $0.20_{-0.07}^{+0.11}$ & $1.10_{-0.04}^{+0.04}$ & $14.05_{-3.01}^{+2.80}$ & 1.01 \\
\hline 4 & & $250-350 a$ & $0.426 \pm 0.003$ & $2.39_{-0.07}^{+0.07}$ & $0.20_{-0.04}^{+0.04}$ & $1.16_{-0.02}^{+0.02}$ & $10.61_{-1.09}^{+1.08}$ & 1.16 \\
\hline 5 & & $250-350 d$ & $0.410 \pm 0.004$ & $2.33_{-0.11}^{+0.11}$ & $0.15_{-0.04}^{+0.05}$ & $1.17_{-0.02}^{+0.02}$ & $13.59_{-1.21}^{+1.17}$ & 1.13 \\
\hline 6 & & $300-400 a$ & $0.428 \pm 0.003$ & $2.33_{-0.11}^{+0.11}$ & $0.15_{-0.04}^{+0.05}$ & $1.17_{-0.02}^{+0.02}$ & $13.59_{-1.21}^{+1.17}$ & 1.13 \\
\hline 7 & & $300-400 d$ & $0.419 \pm 0.004$ & $2.29_{-0.16}^{+0.16}$ & $0.16_{-0.06}^{+0.08}$ & $1.20_{-0.03}^{+0.03}$ & $13.63_{-1.87}^{+1.77}$ & 0.98 \\
\hline 8 & & $350-450$ & $0.421 \pm 0.005$ & $2.23_{-0.55}^{+0.44}$ & $0.13_{-0.10}^{+0.27}$ & $1.31_{-0.08}^{+0.07}$ & $11.74_{-4.60}^{+3.63}$ & 0.87 \\
\hline 1 & O-05-00 & $0-250$ & $0.402 \pm 0.004$ & $2.25_{-0.15}^{+0.15}$ & $0.10_{-0.03}^{+0.05}$ & $1.05_{-0.04}^{+0.04}$ & $16.51_{-2.18}^{+2.60}$ & 1.24 \\
\hline 2 & & $200-300$ & $0.377 \pm 0.005$ & $2.01_{-0.13}^{+0.13}$ & $0.06_{-0.02}^{+0.02}$ & $1.09_{-0.03}^{+0.03}$ & $20.18_{-1.86}^{+2.15}$ & 1.39 \\
\hline 3 & & $250-350 \mathrm{a}$ & $0.394 \pm 0.003$ & $1.92_{-0.10}^{+0.17}$ & $0.05_{-0.01}^{+0.03}$ & $1.05_{-0.05}^{+0.03}$ & $24.65_{-2.96}^{+5.38}$ & 1.30 \\
\hline 4 & & $250-350 \mathrm{~d}$ & $0.374 \pm 0.004$ & $2.03_{-0.14}^{+0.14}$ & $0.07_{-0.02}^{+0.03}$ & $1.12_{-0.03}^{+0.03}$ & $20.71_{-1.65}^{+1.87}$ & 1.80 \\
\hline 5 & & $300-400 a$ & $0.390 \pm 0.003$ & $2.06_{-0.12}^{+0.12}$ & $0.09_{-0.03}^{+0.03}$ & $1.20_{-0.02}^{+0.02}$ & $16.38_{-1.11}^{+1.21}$ & 1.99 \\
\hline 6 & & $300-400 d$ & $0.375 \pm 0.004$ & $2.05_{-0.24}^{+0.23}$ & $0.08_{-0.04}^{+0.06}$ & $1.19_{-0.04}^{+0.04}$ & $19.69_{-1.96}^{+2.25}$ & 1.54 \\
\hline 7 & & $350-450 a$ & $0.381 \pm 0.003$ & $2.24_{-0.23}^{+0.22}$ & $0.15_{-0.07}^{+0.11}$ & $1.20_{-0.04}^{+0.04}$ & $18.34_{-2.63}^{+2.58}$ & 1.26 \\
\hline 8 & & $350-450 \mathrm{~d}$ & $0.375 \pm 0.005$ & $2.34_{-0.39}^{+0.33}$ & $0.17_{-0.11}^{-0.24}$ & $1.26_{-0.05}^{+0.05}$ & $16.23_{-4.38}^{+3.25}$ & 1.22 \\
\hline 9 & & $400-500 a$ & $0.372 \pm 0.005$ & $1.83_{-0.55}^{+0.52}$ & $0.05_{-0.04}^{+0.14}$ & $1.36_{-0.06}^{+0.07}$ & $14.25_{-2.38}^{+2.56}$ & 0.68 \\
\hline 10 & & $400-500 \mathrm{~d}$ & $0.367 \pm 0.008$ & $2.28_{-0.69}^{+0.49}$ & $0.14_{-0.12}^{+0.36}$ & $1.39_{-0.06}^{+0.07}$ & $13.10_{-4.79}^{+3.10}$ & 1.01 \\
\hline 11 & & $450-550$ & $0.392 \pm 0.006$ & $2.23_{-1.84}^{+0.96}$ & $0.08_{-0.08}^{+0.68}$ & $1.49_{-0.09}^{+0.08}$ & $11.65_{-5.67}^{+3.71}$ & 1.17 \\
\hline 1 & O-07-01 & $0-200$ & $0.368 \pm 0.003$ & $2.79_{-0.18}^{+0.17}$ & $0.18_{-0.06}^{+0.09}$ & $1.00_{-0.03}^{+0.03}$ & $11.79_{-2.92}^{+2.55}$ & 0.97 \\
\hline 2 & & $150-250 \mathrm{a}$ & $0.359 \pm 0.003$ & $2.64_{-0.16}^{+0.16}$ & $0.15_{-0.05}^{+0.07}$ & $1.01_{-0.03}^{+0.02}$ & $16.03_{-2.23}^{+2.04}$ & 1.12 \\
\hline 3 & & $150-250 \mathrm{~d}$ & $0.377 \pm 0.005$ & $2.54_{-0.32}^{+0.29}$ & $0.10_{-0.06}^{+0.10}$ & $1.10_{-0.03}^{+0.03}$ & $12.93_{-2.64}^{+1.97}$ & 1.17 \\
\hline 4 & & $200-300 a$ & $0.347 \pm 0.002$ & $2.76_{-0.18}^{+0.17}$ & $0.23_{-0.08}^{+0.12}$ & $1.08_{-0.02}^{+0.02}$ & $14.56_{-2.93}^{+2.45}$ & 1.48 \\
\hline 5 & & $200-300 d$ & $0.358 \pm 0.005$ & $2.28_{-0.57}^{+0.52}$ & $0.05_{-0.04}^{+0.14}$ & $1.20_{-0.03}^{+0.04}$ & $14.34_{-2.89}^{+1.84}$ & 1.03 \\
\hline 6 & & $250-350 \mathrm{a}$ & $0.346 \pm 0.003$ & $2.29_{-0.33}^{+0.30}$ & $0.08_{-0.05}^{+0.09}$ & $1.19_{-0.03}^{+0.03}$ & $15.49_{-2.03}^{+1.68}$ & 1.06 \\
\hline 7 & & $250-350 \mathrm{~d}$ & $0.365 \pm 0.005$ & $3.08_{-0.38}^{+0.30}$ & $0.47_{-0.29}^{+0.39}$ & $1.42_{-0.09}^{+0.13}$ & $6.18_{-2.57}^{+3.47}$ & 1.26 \\
\hline 8 & & $300-400 a$ & $0.364 \pm 0.003$ & $2.76_{-0.30}^{+0.24}$ & $0.30_{-0.16}^{+0.23}$ & $1.35_{-0.05}^{+0.07}$ & $8.83_{-2.82}^{+2.78}$ & 1.13 \\
\hline 9 & & $300-400 d$ & $0.375 \pm 0.004$ & $2.87_{-0.29}^{+0.23}$ & $0.37_{-0.19}^{+0.24}$ & $1.51_{-0.06}^{+0.08}$ & $6.11_{-1.74}^{+2.07}$ & 1.07 \\
\hline 10 & & $350-450 \mathrm{a}$ & $0.376 \pm 0.003$ & $2.77_{-0.20}^{+0.17}$ & $0.33_{-0.13}^{+0.17}$ & $1.42_{-0.03}^{+0.04}$ & $9.39_{-1.79}^{+1.80}$ & 1.01 \\
\hline 11 & & $350-450 \mathrm{~d}$ & $0.396 \pm 0.004$ & $2.72_{-0.18}^{+0.15}$ & $0.30_{-0.11}^{+0.14}$ & $1.51_{-0.03}^{+0.04}$ & $7.30_{-1.26}^{+1.29}$ & 1.09 \\
\hline 12 & & $400-500 a$ & $0.395 \pm 0.003$ & $2.76_{-0.19}^{+0.16}$ & $0.35_{-0.14}^{+0.17}$ & $1.49_{-0.04}^{+0.05}$ & $8.09_{-1.57}^{+1.62}$ & 1.13 \\
\hline 13 & & $400-500 \mathrm{~d}$ & $0.404 \pm 0.004$ & $2.60_{-0.21}^{+0.17}$ & $0.28_{-0.11}^{+0.15}$ & $1.52_{-0.04}^{+0.05}$ & $7.85_{-1.47}^{+1.45}$ & 1.25 \\
\hline 14 & & $450-550$ & $0.413 \pm 0.003$ & $2.67_{-0.37}^{+0.26}$ & $0.35_{-0.22}^{+0.30}$ & $1.54_{-0.06}^{+0.09}$ & $7.73_{-2.47}^{+2.69}$ & 0.96 \\
\hline 1 & O-09-06 & $0-150$ & $0.458 \pm 0.004$ & $3.22_{-0.10}^{+0.12}$ & $0.26_{-0.04}^{+0.05}$ & $1.92_{-0.09}^{+0.09}$ & $0.37_{-0.07}^{+0.09}$ & 1.75 \\
\hline 2 & & $100-200 a$ & $0.457 \pm 0.004$ & $3.18_{-0.12}^{+0.14}$ & $0.27_{-0.05}^{+0.06}$ & $1.92_{-0.07}^{+0.07}$ & $0.66_{-0.10}^{+0.13}$ & 1.51 \\
\hline 3 & & $100-200 d$ & $0.496 \pm 0.005$ & $3.03_{-0.11}^{+0.11}$ & $0.23_{-0.04}^{+0.05}$ & $1.91_{-0.07}^{+0.07}$ & $0.71_{-0.11}^{+0.14}$ & 1.93 \\
\hline 4 & & $150-250 \mathrm{a}$ & $0.484 \pm 0.003$ & $2.97_{-0.25}^{+0.25}$ & $0.19_{-0.08}^{+0.10}$ & $1.79_{-0.09}^{+0.10}$ & $1.37_{-0.33}^{+0.44}$ & 1.19 \\
\hline 5 & & $150-250 \mathrm{~d}$ & $0.500 \pm 0.004$ & $3.10_{-0.16}^{+0.18}$ & $0.28_{-0.07}^{+0.09}$ & $1.95_{-0.07}^{+0.07}$ & $1.09_{-0.16}^{+0.21}$ & 1.71 \\
\hline 6 & & $200-300$ & $0.497 \pm 0.005$ & $3.08_{-0.32}^{+0.39}$ & $0.27_{-0.13}^{+0.20}$ & $1.92_{-0.10}^{+0.11}$ & $1.53_{-0.34}^{+0.49}$ & 1.35 \\
\hline
\end{tabular}

Notes. \# is the label of each rate band of different RXTE observations. Rate bands are all extracted from energy interval 2-60 keV; HR1 is defined as the hardness ratio between the $6.5-15.0 \mathrm{keV}$ and $2.0-6.5 \mathrm{keV}$ count rates; PL index is the powerlaw photon index; NORM powerlaw is the normalization of the powerlaw model; $T_{\mathrm{in}}$ is the inner temperature of the diskbb model in XSPEC; NORM diskbb is the normalization of the diskbb model that is proportional to the square of the inner disk radius. The symbol " $g$ " denotes the energy spectra that a Gaussian was used to fit. The symbol "a" ("d") denotes the ascending (descending) parts of each rate band. 
Table 3. continued

\begin{tabular}{|c|c|c|c|c|c|c|c|c|}
\hline$\#$ & Obs. ID & $\begin{array}{l}\text { Rate band } \\
\text { (cts/s) }\end{array}$ & HR1 & PL index & $\begin{array}{c}\text { NORM } \\
\text { powerlaw }\end{array}$ & $\begin{array}{c}T_{\text {in }} \\
(\mathrm{keV})\end{array}$ & $\begin{array}{l}\text { NORM } \\
\text { diskbb }\end{array}$ & $\chi_{\text {red }}^{2}$ \\
\hline 1 & O-37-04 & $0-200$ & $0.515 \pm 0.014$ & $5.26_{-1.54}^{+2.84}$ & $2.46_{-1.81}^{+2.09}$ & $2.21_{-0.27}^{+0.39}$ & $0.25_{-0.16}^{+0.28}$ & 1.20 \\
\hline 2 & & $150-250 \mathrm{a}$ & $0.517 \pm 0.009$ & $5.09_{-1.05}^{+1.33}$ & $2.80_{-2.11}^{+12.32}$ & $2.11_{-0.19}^{+0.21}$ & $0.78_{-0.44}^{+0.38}$ & $1.15_{g}$ \\
\hline 3 & & $150-250 \mathrm{~d}$ & $0.514 \pm 0.012$ & $4.03_{-0.50}^{+0.92}$ & $0.88_{-0.45}^{+2.00}$ & $2.11_{-0.12}^{+0.11}$ & $0.84_{-0.17}^{+0.23}$ & $0.92_{g}$ \\
\hline 4 & & $200-300 a$ & $0.522 \pm 0.004$ & $4.21_{-0.70}^{+1.28}$ & $1.02_{-0.55}^{+2.96}$ & $2.13_{-0.06}^{+0.06}$ & $0.99_{-0.16}^{+0.22}$ & 1.28 \\
\hline 5 & & $200-300 d$ & $0.522 \pm 0.005$ & $3.81_{-0.53}^{+1.02}$ & $0.67_{-0.32}^{+1.28}$ & $2.10_{-0.08}^{+0.06}$ & $1.25_{-0.17}^{+0.19}$ & 1.09 \\
\hline 6 & & $250-350 \mathrm{a}$ & $0.532 \pm 0.004$ & $3.70_{-0.33}^{+0.52}$ & $0.77_{-0.25}^{+0.56}$ & $2.18_{-0.06}^{+0.04}$ & $1.12_{-0.11}^{+0.12}$ & 1.64 \\
\hline 7 & & $250-350 d$ & $0.535 \pm 0.005$ & $3.74_{-0.34}^{+0.54}$ & $0.82_{-0.27}^{+0.63}$ & $2.17_{-0.05}^{+0.04}$ & $1.23_{-0.11}^{+0.11}$ & 1.62 \\
\hline 8 & & $300-400 a$ & $0.541 \pm 0.003$ & $3.86_{-0.38}^{+0.58}$ & $1.10_{-0.38}^{+0.93}$ & $2.24_{-0.05}^{+0.04}$ & $1.12_{-0.12}^{+0.13}$ & 1.69 \\
\hline 9 & & $300-400 d$ & $0.538 \pm 0.004$ & $3.51_{-0.32}^{+0.55}$ & $0.67_{-0.23}^{+0.54}$ & $2.17_{-0.07}^{+0.05}$ & $1.39_{-0.14}^{+0.17}$ & 1.66 \\
\hline 10 & & $350-450$ & $0.549 \pm 0.005$ & $3.88_{-0.57}^{+1.08}$ & $1.17_{-0.59}^{+2.48}$ & $2.21_{-0.09}^{+0.08}$ & $1.43_{-0.25}^{+0.31}$ & 1.15 \\
\hline 1 & O-08-03 & $0-200$ & $0.430 \pm 0.006$ & $2.01_{-0.28}^{+0.27}$ & $0.03_{-0.02}^{+0.03}$ & $1.05_{-0.07}^{+0.06}$ & $11.24_{-2.02}^{+2.80}$ & 1.19 \\
\hline 2 & & $150-250$ & $0.428 \pm 0.006$ & $2.29_{-0.33}^{+0.30}$ & $0.07_{-0.04}^{+0.08}$ & $1.15_{-0.05}^{+0.05}$ & $9.11_{-1.95}^{+1.52}$ & 1.50 \\
\hline 3 & & $200-300 \mathrm{a}$ & $0.395 \pm 0.003$ & $2.44_{-0.33}^{+0.27}$ & $0.13_{-0.07}^{+0.012}$ & $1.24_{-0.04}^{+0.04}$ & $8.48_{-2.31}^{+1.70}$ & 1.38 \\
\hline 4 & & $200-300 d$ & $0.418 \pm 0.005$ & $2.80_{-0.29}^{+0.23}$ & $0.32_{-0.17}^{+0.20}$ & $1.57_{-0.12}^{+0.17}$ & $2.77_{-1.28}^{+1.83}$ & 0.97 \\
\hline 5 & & $250-350 \mathrm{a}$ & $0.395 \pm 0.003$ & $2.20_{-0.34}^{+0.28}$ & $0.07_{-0.04}^{+0.08}$ & $1.38_{-0.03}^{+0.03}$ & $7.73_{-1.30}^{+0.98}$ & 1.14 \\
\hline 6 & & $250-350 \mathrm{~d}$ & $0.431 \pm 0.005$ & $2.67_{-0.22}^{+0.18}$ & $0.26_{-0.11}^{+0.0 .14}$ & $1.46_{-0.05}^{+0.06}$ & $5.38_{-1.43}^{+1.52}$ & 1.16 \\
\hline 7 & & $300-400 a$ & $0.408 \pm 0.003$ & $2.32_{-0.45}^{+0.30}$ & $0.11_{-0.08}^{+0.13}$ & $1.49_{-0.04}^{+0.03}$ & $6.54_{-1.62}^{+1.28}$ & 1.46 \\
\hline 8 & & $300-400 d$ & $0.440 \pm 0.004$ & $2.40_{-0.36}^{+0.26}$ & $0.14_{-0.09}^{+0.13}$ & $1.47_{-0.03}^{+0.04}$ & $7.46_{-1.59}^{+1.32}$ & 0.86 \\
\hline 9 & & $350-450 \mathrm{a}$ & $0.416 \pm 0.003$ & $2.36_{-0.47}^{+0.31}$ & $0.13_{-0.09}^{+0.15}$ & $1.64_{-0.05}^{+0.07}$ & $5.16_{-1.31}^{+1.17}$ & 0.98 \\
\hline 10 & & $350-450 \mathrm{~d}$ & $0.446 \pm 0.004$ & $2.77_{-0.19}^{+0.16}$ & $0.40_{-0.15}^{+0.17}$ & $1.61_{-0.06}^{+0.07}$ & $4.86_{-1.16}^{+1.37}$ & 0.79 \\
\hline 11 & & $400-500 \mathrm{a}$ & $0.437 \pm 0.003$ & $2.55_{-0.40}^{+0.28}$ & $0.23_{-0.15}^{+0.23}$ & $1.66_{-0.07}^{+0.10}$ & $5.17_{-1.62}^{+1.71}$ & 0.96 \\
\hline
\end{tabular}


Table 4. Spectral parameters for the different rate bands from the six typical RXTE observations, which are fit with diskpbb+powerlaw model.

\begin{tabular}{|c|c|c|c|c|c|c|c|c|c|}
\hline$\#$ & Obs. ID & $\begin{array}{c}\text { Rate band } \\
(\mathrm{cts} / \mathrm{s})\end{array}$ & HR2 & PL index & $\begin{array}{c}\text { NORM } \\
\text { powerlaw }\end{array}$ & $\begin{array}{l}\text { “ } p \text { " parameter } \\
\text { value }\end{array}$ & $\begin{array}{c}T_{\text {in }} \\
(\mathrm{keV})\end{array}$ & $\begin{array}{l}\text { NORM } \\
\text { diskpbb }\end{array}$ & $\chi_{\text {red }}^{2}$ \\
\hline 1 & O-04-03 & $0-250$ & $0.802 \pm 0.011$ & $2.70_{-0.32}^{+0.20}$ & $0.271_{-0.155}^{+0.174}$ & $0.74_{-0.74}^{+0.26}$ & $1.07_{-0.05}^{+0.21}$ & $10.92_{-9.00}^{+3.48}$ & 1.25 \\
\hline 2 & & $200-300 a$ & $0.699 \pm 0.009$ & $2.21_{-0.24}^{+0.21}$ & $0.101_{-0.048}^{+0.077}$ & $0.65_{-0.14}^{+0.35}$ & $1.21_{-0.13}^{+0.18}$ & $7.05_{-4.89}^{+16.09}$ & 1.10 \\
\hline 3 & & $200-300 d$ & $0.708 \pm 0.012$ & $2.26_{-0.37}^{+0.32}$ & $0.103_{-0.061}^{+0.162}$ & $0.49_{-0.06}^{+0.51}$ & $1.32_{-0.23}^{+0.25}$ & $2.60_{-1.78}^{+16.06}$ & 1.00 \\
\hline 4 & & $250-350 a$ & $0.735 \pm 0.008$ & $2.27_{-0.16}^{+0.13}$ & $0.142_{-0.051}^{+0.001}$ & $0.54_{-0.06}^{+0.17}$ & $1.31_{-0.14}^{+0.15}$ & $3.24_{-1.77}^{+5.64}$ & 1.12 \\
\hline 5 & & $250-350 d$ & $0.753 \pm 0.010$ & $1.89_{-0.23}^{+0.21}$ & $0.042_{-0.020}^{+0.034}$ & $0.48_{-0.02}^{+0.04}$ & $1.48_{-0.12}^{+0.13}$ & $1.89_{-0.78}^{+1.53}$ & 0.85 \\
\hline 6 & & $300-400 a$ & $0.677 \pm 0.005$ & $1.99_{-0.26}^{+0.27}$ & $0.070_{-0.037}^{+0.078}$ & $0.49_{-0.04}^{+0.12}$ & $1.48_{-0.21}^{+0.18}$ & $2.01_{-1.05}^{+3.35}$ & 0.76 \\
\hline 7 & & $300-400 d$ & $0.683 \pm 0.007$ & $1.86_{-0.33}^{+0.30}$ & $0.046_{-0.029}^{+0.062}$ & $0.48_{-0.03}^{+0.08}$ & $1.51_{-0.18}^{+0.20}$ & $1.97_{-1.05}^{+2.94}$ & 0.86 \\
\hline 8 & & $350-450$ & $0.591 \pm 0.010$ & $2.23_{-0.70}^{+0.45}$ & $0.123_{-0.079}^{+0.268}$ & $0.75_{-0.24}^{+0.25}$ & $1.32_{-0.08}^{+0.30}$ & $11.65_{-9.49}^{+1.92}$ & 0.89 \\
\hline 1 & O-05-00 & $0-250$ & $0.830 \pm 0.009$ & $2.17_{-0.24}^{+0.21}$ & $0.081_{-0.038}^{+0.060}$ & $0.56_{-0.14}^{+0.12}$ & $1.15_{-0.16}^{+0.13}$ & $6.23_{-3.03}^{+26.08}$ & 1.24 \\
\hline 2 & & $200-300$ & $0.748 \pm 0.007$ & $1.49_{-0.02}^{+0.29}$ & $0.022_{-0.008}^{+0.012}$ & $0.45_{-0.02}^{+0.03}$ & $1.49_{-0.11}^{+0.12}$ & $1.24_{-0.50}^{+0.91}$ & 1.65 \\
\hline 3 & & $250-350 \mathrm{a}$ & $0.729 \pm 0.007$ & $1.43_{-0.20}^{+0.19}$ & $0.014_{-0.006}^{+0.010}$ & $0.44_{-0.01}^{+0.02}$ & $1.68_{-0.13}^{+0.15}$ & $0.78_{-0.31}^{+0.51}$ & 1.20 \\
\hline 4 & & $250-350 \mathrm{~d}$ & $0.745 \pm 0.009$ & $1.46_{-0.28}^{+0.24}$ & $0.014_{-0.008}^{+0.014}$ & $0.45_{-0.02}^{+0.03}$ & $1.52_{-0.13}^{+0.16}$ & $1.59_{-0.78}^{+1.49}$ & 1.40 \\
\hline 5 & & $300-400 a$ & $0.650 \pm 0.006$ & $1.43_{-0.26}^{+0.24}$ & $0.014_{-0.008}^{+0.014}$ & $0.47_{-0.02}^{+0.03}$ & $1.63_{-0.13}^{+0.15}$ & $1.39_{-0.59}^{+1.04}$ & 1.47 \\
\hline 6 & & $300-400 d$ & $0.653 \pm 0.009$ & $1.59_{-0.43}^{+0.40}$ & $0.020_{-0.014}^{+0.042}$ & $0.50_{-0.05}^{+0.10}$ & $1.44_{-0.17}^{+0.19}$ & $3.50_{-1.95}^{+6.37}$ & 1.46 \\
\hline 7 & & $350-450 a$ & $0.569 \pm 0.007$ & $1.70_{-0.53}^{+0.46}$ & $0.032_{-0.025}^{+0.081}$ & $0.49_{-0.04}^{+0.11}$ & $1.52_{-0.23}^{+0.26}$ & $2.53_{-1.61}^{+6.23}$ & 1.20 \\
\hline 8 & & $350-450 \mathrm{~d}$ & $0.560 \pm 0.010$ & $1.60_{-0.95}^{+0.76}$ & $0.021_{-0.018}^{+0.073}$ & $0.50_{-0.05}^{+0.16}$ & $1.57_{-0.29}^{+0.10}$ & $2.70_{-1.91}^{+10.02}$ & 1.19 \\
\hline 9 & & $400-500 a$ & $0.486 \pm 0.010$ & $1.36_{-0.79}^{+0.79}$ & $0.012_{-0.011}^{+0.081}$ & $0.56_{-0.07}^{+0.36}$ & $1.57_{-0.24}^{+0.27}$ & $4.16_{-2.80}^{+9.38}$ & 0.66 \\
\hline 10 & & $400-500 \mathrm{~d}$ & $0.479 \pm 0.013$ & $1.82_{-1.20}^{+0.93}$ & $0.038_{-0.036}^{+0.444}$ & $0.59_{-0.10}^{+0.41}$ & $1.54_{-0.21}^{+0.32}$ & $5.51_{-4.02}^{+12.67}$ & 1.02 \\
\hline 11 & & $450-550$ & $0.397 \pm 0.012$ & $2.30_{-1.49}^{+0.73}$ & $0.095_{-0.094}^{+0.873}$ & $0.79_{-0.29}^{+0.21}$ & $1.47_{-0.14}^{+0.42}$ & $13.26_{-11.59}^{+18.07}$ & 1.19 \\
\hline 1 & O-07-01 & $0-200$ & $1.144 \pm 0.012$ & $2.37_{-0.41}^{+0.37}$ & $0.059_{-0.042}^{+0.101}$ & $0.44_{-0.04}^{+0.12}$ & $1.29_{-0.21}^{+0.22}$ & $1.28_{-0.81}^{+3.88}$ & 0.91 \\
\hline 2 & & $150-250 \mathrm{a}$ & $1.029 \pm 0.009$ & $1.94_{-0.35}^{+0.31}$ & $0.022_{-0.014}^{+0.029}$ & $0.43_{-0.02}^{+0.03}$ & $1.41_{-0.14}^{+0.16}$ & $1.03_{-0.52}^{+1.14}$ & 0.79 \\
\hline 3 & & $150-250 \mathrm{~d}$ & $1.053 \pm 0.016$ & $1.72_{-0.56}^{+0.50}$ & $0.011_{-0.009}^{+0.031}$ & $0.47_{-0.03}^{+0.06}$ & $1.41_{-0.15}^{+0.18}$ & $1.59_{-0.89}^{+1.95}$ & 1.03 \\
\hline 4 & & $200-300 \mathrm{a}$ & $0.911 \pm 0.008$ & $1.99_{-0.38}^{+0.36}$ & $0.028_{-0.019}^{+0.047}$ & $0.44_{-0.02}^{+0.04}$ & $1.43_{-0.14}^{+0.15}$ & $1.46_{-0.69}^{+0.84}$ & 1.24 \\
\hline 5 & & $200-300 d$ & $0.899 \pm 0.014$ & $1.53_{-0.77}^{+0.77}$ & $0.007_{-0.006}^{+0.047}$ & $0.53_{-0.06}^{+0.14}$ & $1.39_{-0.15}^{+0.16}$ & $3.75_{-2.14}^{+6.68}$ & 0.98 \\
\hline 6 & & $250-350 \mathrm{a}$ & $0.705 \pm 0.007$ & $1.62_{-0.54}^{+0.49}$ & $0.012_{-0.009}^{+0.035}$ & $0.51_{-0.04}^{+0.08}$ & $1.42_{-0.14}^{+0.16}$ & $3.18_{-1.69}^{+2.21}$ & 0.96 \\
\hline 7 & & $250-350 \mathrm{~d}$ & $0.679 \pm 0.011$ & $3.08_{-1.18}^{+0.30}$ & $0.467_{-0.455}^{+0.396}$ & $0.74_{-0.27}^{+0.22}$ & $1.42_{-0.10}^{+0.32}$ & $6.01_{-5.00}^{+3.41}$ & 1.28 \\
\hline 8 & & $300-400 a$ & $0.532 \pm 0.006$ & $2.84_{-0.43}^{+0.21}$ & $0.378_{-0.265}^{+0.095}$ & $0.75_{-0.19}^{+0.25}$ & $1.30_{-0.04}^{+0.19}$ & $16.16_{-12.67}^{+5.80}$ & 1.14 \\
\hline 9 & & $300-400 d$ & $0.523 \pm 0.007$ & $2.52_{-1.85}^{+0.63}$ & $0.132_{-0.132}^{+0.606}$ & $0.58_{-0.09}^{+0.42}$ & $1.62_{-0.22}^{+0.29}$ & $3.04_{-1.97}^{+11.23}$ & 1.09 \\
\hline 10 & & $350-450 \mathrm{a}$ & $0.467 \pm 0.007$ & $2.88_{-0.30}^{+0.15}$ & $0.447_{-0.159}^{+0.182}$ & $0.77_{-0.14}^{+0.23}$ & $1.37_{-0.04}^{+0.12}$ & $17.16_{-11.38}^{+44.87}$ & 1.01 \\
\hline 11 & & $350-450 \mathrm{~d}$ & $0.451 \pm 0.008$ & $2.81_{-0.50}^{+0.05}$ & $0.300_{-0.111}^{+0.134}$ & $0.75_{-0.17}^{+0.25}$ & $1.45_{-0.04}^{+0.17}$ & $12.47_{-9.06}^{+2.96}$ & 1.10 \\
\hline 12 & & $400-500 \mathrm{a}$ & $0.415 \pm 0.004$ & $2.76_{-0.29}^{+0.17}$ & $0.350_{-0.203}^{+0.170}$ & $0.75_{-0.15}^{+0.25}$ & $1.49_{-0.04}^{+0.11}$ & $8.04_{-4.38}^{+1.64}$ & 1.15 \\
\hline 13 & & $400-500 \mathrm{~d}$ & $0.405 \pm 0.005$ & $2.60_{-0.38}^{+0.17}$ & $0.276_{-0.120}^{+0.150}$ & $0.75_{-0.16}^{+0.25}$ & $1.52_{-0.04}^{+0.12}$ & $7.82_{-4.14}^{+1.45}$ & 1.27 \\
\hline 14 & & $450-550$ & $0.378 \pm 0.006$ & $2.32_{-1.42}^{+0.68}$ & $0.126_{-0.122}^{+0.1283}$ & $0.60_{-0.05}^{+0.10}$ & $1.64_{-0.22}^{+0.04}$ & $4.12_{-2.70}^{+14.50}$ & 0.98 \\
\hline 1 & O-09-06 & $0-150$ & $0.721 \pm 0.007$ & $3.04_{-0.18}^{+0.24}$ & $0.119_{-0.034}^{+0.127}$ & $0.46_{-0.46}^{+0.23}$ & $2.21_{-0.26}^{+0.14}$ & $0.10_{-0.02}^{+0.04}$ & 1.72 \\
\hline 2 & & $100-200$ & $0.551 \pm 0.006$ & - & - & - & - & - & - \\
\hline 3 & & $150-250 \mathrm{a}$ & $0.415 \pm 0.004$ & $0.79_{-0.79}^{+2.41}$ & $0.000_{-0.000}^{+0.030}$ & $0.53_{-0.03}^{+0.47}$ & $2.07_{-0.40}^{+0.15}$ & $0.43_{-0.17}^{+0.75}$ & 1.19 \\
\hline 4 & & $150-250 \mathrm{~d}$ & $0.412 \pm 0.004$ & - & - & $0.50_{-0.01}^{+0.01}$ & $2.42_{-0.07}^{+0.07}$ & $0.21_{-0.04}^{+0.05}$ & 1.80 \\
\hline 5 & & $200-300$ & $0.328 \pm 0.005$ & - & - & $0.53_{-0.02}^{+0.03}$ & $2.29_{-0.10}^{+0.11}$ & $0.38_{-0.11}^{+0.16}$ & 1.38 \\
\hline 1 & O-37-04 & $0-200$ & $0.730 \pm 0.029$ & - & - & - & - & - & - \\
\hline 2 & & $150-250$ & $0.565 \pm 0.013$ & - & - & $0.53_{-0.04}^{+0.05}$ & $2.29_{-0.18}^{+0.21}$ & $0.31_{-0.15}^{+0.28}$ & $1.19_{g}$ \\
\hline 3 & & $200-300 \mathrm{a}$ & $0.482 \pm 0.005$ & - & - & $0.52_{-0.02}^{+0.02}$ & $2.39_{-0.11}^{+0.12}$ & $0.27_{-0.08}^{+0.11}$ & 1.30 \\
\hline 4 & & 200-300d & $0.480 \pm 0.006$ & - & - & $0.53_{-0.02}^{+0.02}$ & $2.33_{-0.09}^{+0.10}$ & $0.40_{-0.11}^{+0.15}$ & 1.05 \\
\hline 5 & & $250-350 \mathrm{a}$ & $0.414 \pm 0.005$ & - & - & $0.52_{-0.01}^{+0.01}$ & $2.47_{-0.07}^{+0.07}$ & $0.31_{-0.06}^{+0.07}$ & 1.59 \\
\hline 6 & & $250-350 d$ & $0.411 \pm 0.005$ & - & - & $0.52_{-0.01}^{+0.01}$ & $2.44_{-0.06}^{+0.06}$ & $0.36_{-0.06}^{+0.08}$ & 1.63 \\
\hline
\end{tabular}

Notes. $T_{\text {in }}$ is the inner temperature of the diskpbb model in XSPEC; NORM diskpbb is the normalization of the diskpbb model that is proportional to the square of the inner disk radius. The other symbols follow the same criteria as in Table 3. 
Table 4. continued.

\begin{tabular}{|c|c|c|c|c|c|c|c|c|c|}
\hline$\#$ & Obs. ID & $\begin{array}{l}\text { Rate band } \\
\text { (cts/s) }\end{array}$ & HR2 & PL index & $\begin{array}{c}\text { NORM } \\
\text { powerlaw }\end{array}$ & $\begin{array}{l}\text { " } p " \text { parameter } \\
\text { value }\end{array}$ & $\begin{array}{c}T_{\text {in }} \\
(\mathrm{keV})\end{array}$ & $\begin{array}{l}\text { NORM } \\
\text { diskpbb }\end{array}$ & $\chi_{\text {red }}^{2}$ \\
\hline 7 & \multirow{17}{*}{ O-08-03 } & $300-400 a$ & $0.362 \pm 0.004$ & - & - & $0.51_{-0.01}^{+0.01}$ & $2.55_{-0.16}^{+0.16}$ & $0.29_{-0.06}^{+0.07}$ & 1.70 \\
\hline 8 & & $300-400 d$ & $0.365 \pm 0.004$ & - & - & $0.53_{-0.01}^{+0.01}$ & $2.44_{-0.07}^{+0.07}$ & $0.44_{-0.09}^{+0.11}$ & 1.65 \\
\hline 9 & & $350-450$ & $0.330 \pm 0.005$ & - & - & $0.52_{-0.02}^{+0.03}$ & $2.48_{-0.13}^{+0.24}$ & $0.41_{-0.14}^{+0.20}$ & 1.10 \\
\hline 1 & & $0-200$ & $1.090 \pm 0.017$ & $1.16_{-0.56}^{+0.46}$ & $0.003_{-0.002}^{+0.005}$ & $0.40_{-0.40}^{+0.04}$ & $1.63_{-0.32}^{+0.32}$ & $3.07_{-0.94}^{+3.69}$ & 0.97 \\
\hline 2 & & $150-250$ & $0.905 \pm 0.015$ & $0.59_{-0.55}^{+0.56}$ & $0.001_{-0.001}^{+0.002}$ & $0.43_{-0.02}^{+0.02}$ & $1.96_{-0.15}^{+0.21}$ & $0.22_{-0.13}^{+0.18}$ & 0.97 \\
\hline 3 & & $200-300 a$ & $0.683 \pm 0.006$ & $0.94_{-0.64}^{+0.62}$ & $0.002_{-0.001}^{+0.009}$ & $0.46_{-0.02}^{+0.03}$ & $1.80_{-0.21}^{+0.22}$ & $0.62_{-0.32}^{+0.72}$ & 1.09 \\
\hline 4 & & $200-300 d$ & $0.729 \pm 0.009$ & $2.86_{-1.31}^{+0.20}$ & $0.385_{-0.361}^{+0.198}$ & $1.00_{-0.53}^{+0.00}$ & $1.52_{-0.12}^{+0.60}$ & $2.76_{-2.30}^{+1.82}$ & 0.99 \\
\hline 5 & & $250-350 a$ & $0.599 \pm 0.006$ & $1.58_{-0.68}^{+0.68}$ & $0.001_{-0.000}^{+0.052}$ & $0.56_{-0.05}^{+0.19}$ & $1.58_{-0.21}^{+0.25}$ & $2.49_{-1.32}^{+5.03}$ & 1.12 \\
\hline 6 & & $250-350 d$ & $0.642 \pm 0.008$ & $1.62_{-0.89}^{+0.93}$ & $0.001_{-0.000}^{+0.177}$ & $0.51_{-0.03}^{+0.08}$ & $1.76_{-0.25}^{+0.21}$ & $1.32_{-0.64}^{+2.45}$ & 1.12 \\
\hline 7 & & $300-400 a$ & $0.517 \pm 0.005$ & $1.11_{-1.05}^{+0.94}$ & $0.003_{-0.003}^{+0.019}$ & $0.55_{-0.05}^{+0.09}$ & $1.78_{-0.21}^{+0.12}$ & $1.76_{-0.95}^{+2.12}$ & 1.41 \\
\hline 8 & & $300-400 d$ & $0.533 \pm 0.006$ & $1.44_{-0.84}^{+0.87}$ & $0.008_{-0.008}^{+0.095}$ & $0.56_{-0.04}^{+0.11}$ & $1.70_{-0.20}^{+0.17}$ & $2.39_{-1.14}^{+3.51}$ & 0.82 \\
\hline 9 & & $350-450 \mathrm{a}$ & $0.442 \pm 0.005$ & $2.12_{-1.43}^{+0.53}$ & $0.063_{-0.061}^{+0.212}$ & $0.66_{-0.12}^{+0.20}$ & $1.70_{-0.11}^{+0.27}$ & $3.67_{-2.31}^{+2.36}$ & 1.00 \\
\hline 10 & & $350-450 \mathrm{~d}$ & $0.432 \pm 0.005$ & $1.53_{-1.16}^{+1.32}$ & $0.010_{-0.010}^{+0.020}$ & $0.52_{-0.03}^{+0.00}$ & $1.90_{-0.30}^{+0.18}$ & $1.41_{-0.56}^{+3.01}$ & 0.76 \\
\hline 11 & & $400-500 a$ & $0.364 \pm 0.007$ & $2.68_{-1.08}^{+0.26}$ & $0.349_{-0.333}^{+0.245}$ & $0.98_{-0.42}^{+0.02}$ & $1.59_{-0.08}^{+0.32}$ & $9.18_{-7.57}^{+3.81}$ & 0.97 \\
\hline 12 & & $400-500 d$ & $0.349 \pm 0.008$ & $2.83_{-1.48}^{+0.26}$ & $0.399_{-0.395}^{+0.316}$ & $0.69_{-0.18}^{+0.31}$ & $1.79_{-0.10}^{+0.11}$ & $3.23_{-2.24}^{+1.97}$ & 0.92 \\
\hline 13 & & $450-550$ & $0.319 \pm 0.004$ & $2.61_{-1.17}^{+0.30}$ & $0.263_{-0.255}^{+0.282}$ & $0.74_{-0.20}^{+0.26}$ & $1.62_{-0.06}^{+0.26}$ & $6.92_{-4.82}^{+17.44}$ & 0.88 \\
\hline 14 & & $500-600$ & $0.457 \pm 0.003$ & - & - & - & - & - & - \\
\hline
\end{tabular}

